\title{
Theoretical and Experimental Study on Normal Contact Stiffness of Plane Joint Surfaces with Surface Texturing
}

\section{Chaochao Yin}

HFUT: Hefei University of Technology

Haihong Huang ( $\square$ huanghaihong@hfut.edu.cn )

Hefei University of Technology

Dan Zhou

HFUT: Hefei University of Technology

Zhifeng Liu

HFUT: Hefei University of Technology

Original Article

Keywords: Surface texturing , Normal contact stiffness , Fractal theory , Structure function method

Posted Date: September 16th, 2021

DOl: https://doi.org/10.21203/rs.3.rs-880073/v1

License: (1) This work is licensed under a Creative Commons Attribution 4.0 International License. Read Full License 


\section{Title page}

\section{Theoretical and Experimental Study on Normal Contact Stiffness of Plane Joint Surfaces} with Surface Texturing

Chao-Chao Yin, is currently a PhD candidate at Key Laboratory of Green Design and Manufacturing of Mechanical Industry, Hefei University of Technology, Hefei, China.

Tel:+86-18297925175; E-mail:2016110111@mail.hfut.edu.cn

Hai-Hong Huang, is currently a professor, PhD Tutor at School of Mechanical Engineering, Hefei University of Technology, Hefei, China.

Tel:+86-13955138301; E-mail: huanghaihong@hfut.edu.cn

Dan Zhou, is currently an associate professor at School of Mechanical Engineering, Hefei University of Technology, Hefei, China.

E-mail: zhoudan80@126.com

Zhi-Feng Liu, is currently a professor, PhD Tutor at School of Mechanical Engineering, Hefei University of Technology, Hefei, China.

E-mail: zhfliuhfut@126.com

Corresponding author: Hai-hong Huang E-mail: huanghaihong@hfut.edu.cn 


\title{
Theoretical and Experimental Study on Normal Contact Stiffness of Plane Joint
}

\author{
Surfaces with Surface Texturing
}

\author{
Chao-Chao Yin ${ }^{1,2} \cdot$ Hai-Hong Huang ${ }^{1,2} \cdot$ Dan Zhou ${ }^{1,2} \bullet$ Zhi-Feng Liu ${ }^{1,2}$
}

\begin{abstract}
Effects of surface texturing on the normal contact stiffness of joint surfaces had been investigated by experiments in many previous researches; however, there are relatively few theoretical models in this regard. The rough surface with surface texturing can be divided into two parts: the textured zone and the remaining zone, and their theoretical models are established respectively in this research considering surface morphology and material properties. For the textured zone, micro textures are modeled theoretically based on the three-dimensional topographic data obtained via a VK-X250 type laser profilometer from KEYENCE. For the remaining zone, the model of normal contact stiffness is established based on the fractal theory for the surface topography description and elastoplastic deformation of surface asperities, and the structure function method is used to calculate the fractal dimension of rough surface profiles. In the experiment, the normal contact stiffness of specimens is obtained under different normal loads, and the test results are compared with the theoretical predictions. The result shows that the predictions of proposed theoretical model are in good agreement with the experimental data. For the joint surfaces with $\mathrm{Sa}>2.69$ $\mu \mathrm{m}$, the normal contact stiffness can be effectively increased through proper surface texturing.
\end{abstract}

Keywords: Surface texturing $\bullet$ Normal contact stiffness $\bullet$ Fractal theory $\bullet$ Structure function method

\section{Introduction}

Plane joint surfaces exist extensively in mechanical structures, such as the fixed joint of machine tool, aeroengine and flange etc. The micro topography of joint surfaces is one of the most important factors that determine not only the tribological properties but also the dynamic behavior of contacts. As one of the important parameters of contact properties, the contact stiffness of joint surfaces has a significant influence on the overall static and dynamic characteristics of mechanical systems under combined loads.

The enhancement of the normal contact stiffness of the

Hai-Hong Huang

huanghaihong@hfut.edu.cn

1 School of Mechanical Engineering, Hefei University of Technology, joint surfaces can reduce the deformation, thereby improving the machining quality of machine tool and the dynamic connection properties of flange [1]. However, it has always been very difficult to accurately predict the contact characteristics of the joint surfaces. The improvement of accuracy for the contact stiffness model is the basis for further development of the machine dynamics modeling and analysis [2-4].

Scholars have been working to establish a reasonable theoretical model of normal contact stiffness. Greenwood and Williamson [5] established a statistical model to simulate the contact stiffness of joint surfaces for the first time, which was called G-W model for short. This research provides a theoretical basis for the study of contact mechanisms of rough surface. On the other hand, Majumbar and Bhushan [6] proposed a theoretical model (M-B model) from the perspective of fractal geometry, which considered the contact characteristics of joint surfaces, and it also solved the problem of scale dependence in statistical models. Based on the above work, the research on the contact characteristics of joint surfaces has been further improved, such as considering multifractal domain [7], elastic-plastic deformation characteristics [8], surface processing methods [9], surface finish [10], lubrication [11] and so on. Also, the research on normal contact stiffness of joint surfaces is gradually developed. Ciavarella et al. [12] improved the G-W model, and modified the relationship between contact load and contact deformation through considering the interaction of asperities. Considering the influence of asymmetry and peak value on rough contact performance, Belhadjamor et al. [13] proposed a numerical model of normal contact stiffness by calculating the actual area and the number of contact asperities. Wang et al. [14] proposed a fractal model of normal contact stiffness based on the elastic deformation characteristics of a single asperity under partial load. Based on the elastic-plastic deformation mechanism of a single pair of contact asperities, Zhang et

Tunxi Road 193, Hefei, 230009,China

2 Key Laboratory of Green Design and Manufacturing of Mechanical Industry, Hefei University of Technology, Hefei, 230009, China 
al. [15] proposed a normal contact stiffness model using fractal model to describe the surface morphology. Essentially, the contact properties analysis of joint surfaces is closely related to the contact characteristics of micro morphology between the two rough surfaces, and the morphology of rough surfaces greatly affects the physical properties of joint surfaces [16-17]. Therefore, it is of great significance to establish an accurate normal contact stiffness model from the perspective of micro morphology.

In recent years, laser micromachining technique has gradually become a research hotspot due to good surface modification function $[18,19]$. Contact properties of joint surfaces can be improved or modified by generating micro textures using laser micromachining technique, which can change the micro morphology of rough surface significantly. Micro textures in the form of circular dimples can effectively reduce friction and wear due to the reduction of contact area, the increase of material hardness of cladding layer like craters and the storage capacity to abrasive particles [20]. The increase of material hardness can enhance the ability of joint surfaces to resist deformation, which is the main factor of influence that surface texturing on the normal contact stiffness of the joint surfaces. Surface texturing refers to the micro structures with regular arrangement on the surface of structures through active design and manufacturing. Because the surface texturing changes the morphology and material properties of rough surface, it has a significant impact on the normal contact stiffness of joint surfaces.

To date, several researches concerned the influence of surface texturing on normal contact stiffness of joint surfaces. M. Arghir [21] proposed a theoretical approach for the analysis of the normal contact stiffness of the sealing ring with surface texturing through simulation. The comparison between the theoretical normal contact stiffness and the experimental data indicated that the estimation model was appropriate, and the accuracy of the model predictions was improved by using the modified bulk-flow model. The research of S. Medina [22] showed that the density of surface asperities can be controlled by altering the distance between the rough surfaces, so as to control the normal contact stiffness of joint surfaces. Z. Fuadi [23] proposed an experimental method to study the influence of asperity density on the tangent contact stiffness by machining the micro texture like pyramid on the surfaces of specimens. The research showed that the higher the contact density is, the greater the tangent contact stiffness is, and a power is proposed to explain the relationship between the certain eigenmode frequency of testing system and the tangential contact stiffness. Zhang Yi [24] designed an experimental platform to test the contact stiffness and contact damping of mechanical joint surfaces, and studied the influence of the grooved surface texturing on the contact properties. The results showed that the surface texturing could reduce the contact stiffness and increase the contact damping. The above results show that the surface texturing has a significant effect on the contact stiffness of the joint surfaces.

The previous researches mainly studied the influence of surface texturing on the contact stiffness of joint surfaces through simulations and experiments. However, there are few theoretical models, and the impact mechanism of surface texturing on the normal contact stiffness of joint surfaces is still unclear, which needs further investigation. This research focuses on the changes in micromorphology and material properties of rough surfaces, and proposes a theoretical calculation method for the normal contact stiffness of joint surfaces with surface texturing. The theoretical model is verified by experimentally measuring the normal contact stiffness.

\section{Theoretical model}

\subsection{Model assumptions}

\subsubsection{Surface topography}

Contact stiffness is mainly used to describe the contact characteristics of two elastic rough surfaces. Previous studies [25] showed that the contact between two rough surfaces can be equivalent to that of a rough surface and a rigid smooth surface, so the same can be done for the contact between a textured surface and a rough surface, as shown in Figure 1. Because the micro textures occupy only a part of the entire contact surfaces, the textured surface can be divided into the textured zone and the remaining zone, as shown in Figure 1.
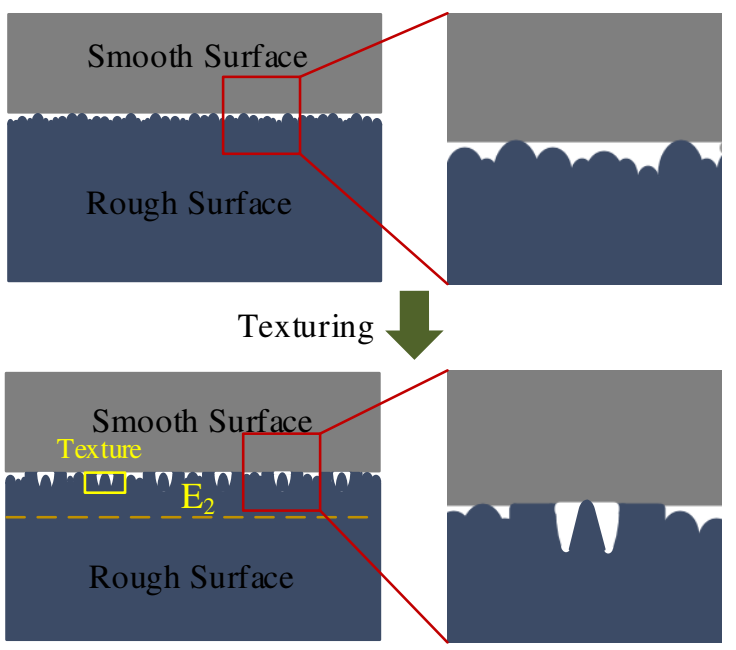

Figure 1 Schematic diagram of contact between rough surface and rigid smooth surface 
The micro textures only change the morphology of textured zone, but has almost no effect on the morphology of remaining zone. Therefore, the surface morphology of remaining zone is similar to that of specimens before fabricating the surface texture. Regardless of the differences among micro textures, it is assumed that the three-dimensional morphology of each texture is analogous, as shown in Figure 2.

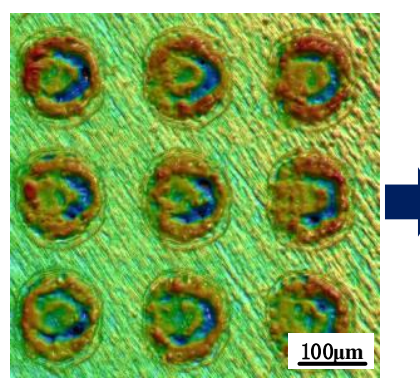

Remaining zone

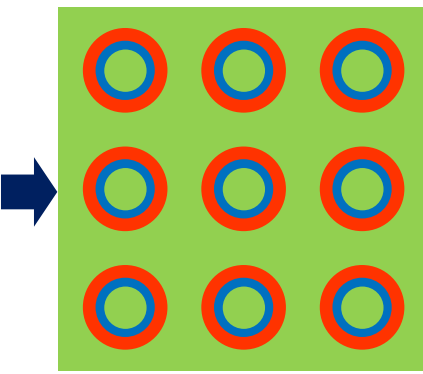

Textured zone
Figure 2 Region division of the textured surface

\subsubsection{Microstructure}

The profile of textured specimen was obtained by wire cutting and etched with $4 \%$ nital, as shown in figure 3. In the textured zone, laser processing causes surface materials to melt rapidly. With the increase of distance from the surface, the absorbed laser energy decreases, forming high temperature gradient. In the region near the texture, the temperature exceeds $\mathrm{Ac}_{1}$, martensite and troostite [26,27] are produced. Otherwise, the temperature does not reach $\mathrm{Ac}_{1}$ and there is not phase transformation, but the grain size is refined and the dislocation density is increased. The heat affected zone is formed with a thickness of about $100 \mu \mathrm{m}$. The elastic modulus $E_{2}$ of material in textured zone is regarded as an invariant constant.

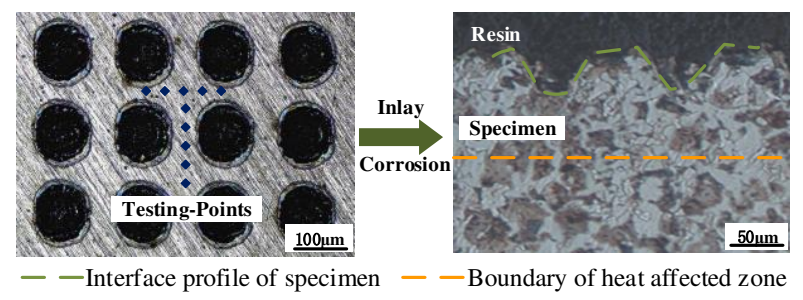

Figure 3 The effect of laser processing on microstructure

The surface hardness and the hardness along the depth direction of texture are measured by microhardness tester. The load is $0.25 \mathrm{~N}$, and the holding time is $15 \mathrm{~s}$. The distance between the testing points is $30 \mu \mathrm{m}$, and the diagonal length of indentation is $10-20 \mu \mathrm{m}$. The results are the average of 5 points. The experimental results show that the surface hardness of remaining original area is about $200 \mathrm{HV}_{0.025}$, which is consistent with that of the original material. Along the depth direction of texture, it can be seen from Figure 4 that the hardness of material decreases gradually, and the maximum value is $290 \mathrm{HV}_{0.025}$. When the depth reaches to $100 \mu \mathrm{m}$, the hardness is stable at about $200 \mathrm{HV}_{0.025}$.

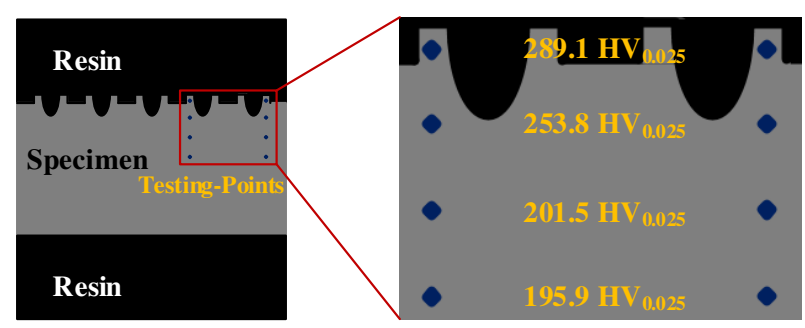

Figure 4 The schematic of hardness test

The normal contact stiffness $\mathrm{K}$ of the entire joint surfaces is synthesized by that of textured zone and remaining zone. The theoretical model of normal contact stiffness $\mathrm{K}$ can be expressed as follows:

$$
K=\left\{\begin{array}{ll}
K_{2} & \delta \leq H-d \\
K_{1}+K_{2} & \delta>H-d
\end{array},\right.
$$

where $K_{1}$ is the normal contact stiffness of the remaining zone, $K_{2}$ is the normal contact stiffness of the textured zone, $H$ is the average height of the textures, $d$ is the average height of surface asperities, and $\delta$ is deformation of surface texture.

\subsection{Normal contact stiffness of the remaining zone}

On the basis of KE model, the influence of interaction between asperities on the normal contact stiffness is considered by introducing the fractal dimension $D(1<D<2)$ of rough surface profiles [28]. Based on the fractal model, the normal contact stiffness $K_{0}$ of joint surfaces can be expressed as follows [29]:

$$
K_{0}=\int_{a_{c}}^{a_{l}} k \cdot n(a) d a,
$$

where $K_{0}$ is the normal contact stiffness of the joint surfaces before processing the surface texture, $a_{\mathrm{c}}$ is the critical contact area, $a_{1}$ is the actual contact area of the largest asperity, and $k$ is normal contact stiffness of a single asperity. 
According to fractal contact theory [30], the critical contact area of elastoplastic deformation can be expressed as

$$
a_{c}=G^{2}\left(\frac{2 E}{H_{T}}\right)^{\frac{2}{D-1}} \text {, }
$$

where HT is hardness of textured surface, and G is fractal roughness.

For a single asperity, the normal contact stiffness $k$ can be expressed as [31]

$$
k=2 E \sqrt{\frac{3 \mathrm{a}}{16}},
$$

where a is the contact area of the asperity, and $E$ is equivalent elastic modulus.

According to Hertz contact theory, the equivalent elastic modulus $E$ can be obtained by the elastic modulus and Poisson ratios of the jointed materials.

$$
E=\left(\frac{1-v_{u}^{2}}{E_{u}}+\frac{1-v_{l}^{2}}{E_{l}}\right)^{-1},
$$

where $V_{\mathrm{u}}$ and $V_{1}$ are the Poisson ratios of the two rough surfaces, respectively, $E_{\mathrm{u}}$ and $E_{1}$ are the elastic moduli of the two rough surfaces, respectively.

Based on the statistical principle, the probability distribution function $n(a)$ of the truncated micro-contact area $a$ can be given by [13]

$$
n(a)=\frac{D}{2} \psi^{\frac{2-D}{2}}\left(a_{l}^{\prime}\right)^{\frac{D}{2}}(a)^{-\left(\frac{D+2}{2}\right)}
$$

Notice that $a_{l}^{\prime}$ is the cross-sectional area of the maximum contact point, $\psi$ is the domain expansion factor of the contact size distribution of asperities, and $D$ is the fractal dimension of rough surface profile.

The domain expansion factor $\psi$ is determined by the fractal dimension $D$, and the relationship between $\psi$ and $D$ can be expressed as [7]

$$
\psi^{\frac{2-D}{2}}-\left(1+\psi^{\frac{-D}{2}}\right)^{\frac{D-2}{2}}=\frac{2-D}{D}
$$

Introducing Eqs. (4) and (6) into Eq. (2), the normal contact stiffness $K_{0}$ of joint surfaces can be given by

$$
K_{0}=\frac{\sqrt{6} E D}{4(1-D)} \psi^{\frac{2-D}{2}} a_{l}^{\frac{D}{2}}\left(a_{l}^{\frac{1-D}{2}}-a_{c}^{\frac{1-D}{2}}\right),
$$

According to Eq. (8), it can be concluded that the normal contact stiffness of joint surfaces is directly related to the contact area $a_{1}$ of the largest asperity. For a given surface, the relationship between the critical contact area $a_{\mathrm{c}}$ and the actual contact area $a_{1}$ of the largest asperity can be expressed as follows:

$$
\begin{aligned}
& a_{l}^{\frac{1-D}{2}}=\lambda a_{\mathrm{c}}^{\frac{1-D}{2}}, \\
& \lambda=100^{(1-D) / 2},
\end{aligned}
$$

Therefore, the normal contact stiffness $K_{0}$ can be expressed as a function of actual contact area of the largest asperity [25], and Eq. (8) can be simplified as follows:

$$
\begin{gathered}
K_{0}=\frac{\alpha E D}{1-D} \psi^{\frac{2-D}{2}} a_{l}^{\frac{1}{2}}, \\
\alpha=3 \sqrt{3}\left(1-\lambda^{-1}\right),
\end{gathered}
$$

The normal contact stiffness is essentially the elastoplastic deformation of asperities interaction, which is mainly related to the surface morphology and material properties. For the remaining zone, the surface morphology is almost not affected by surface texturing, and the surface hardness and elastic modulus remain unchanged. As a result, the theoretical model of normal contact stiffness is the same as that of before surface texturing. The normal contact stiffness $K_{0}$ of the specimens before texturing is obtained by experiment. And the normal contact stiffness $K_{1}$ of remaining zone can be expressed as follows:

$$
K_{1}=b K_{0},
$$

where $b$ is the ratio coefficient of the remaining zone area to the apparent contact area.

In order to obtain the ratio coefficient $b$, it is necessary to accurately get the area of textured zone. Nine sampled textures are selected by the cross-sampling method, as shown in Figure 5. The sampled textures are selected with equal spacing in two directions perpendicular to each other. According to the data of surface topography, the bottom 
diameter of each texture can be obtained. It is assumed that the average value is the bottom diameter of all the textures, and the area of the textured zone can then be calculated, as well as the coefficient $b$.

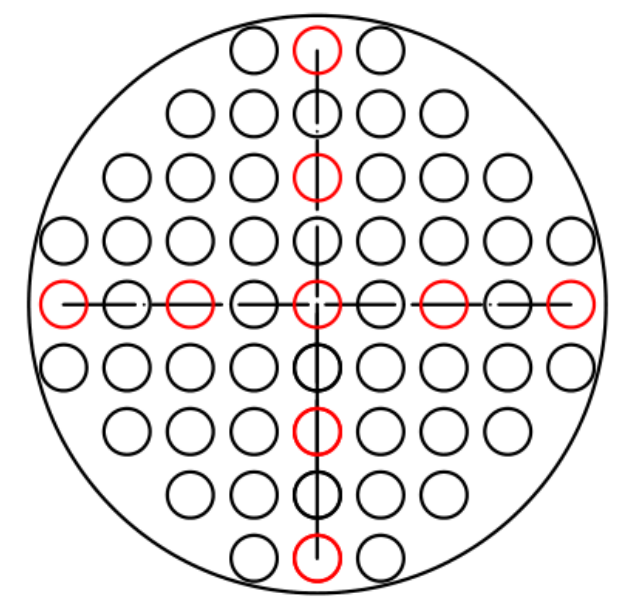

Figure 5 Schematic diagram of texture sample selection

\subsection{Measurement of the fractal dimension of rough surface profiles}

The topography of rough surface can be objectively characterized by fractal geometry theory. The rough surfaces of the specimens can be considered to be isotropic. The structure function method is used to calculate the fractal dimension of rough surface profiles. The incremental variance of a specific rough surface contour curve is defined as the structure function of the curve. The structure function $S(\tau)$ can be expressed in the form [32]:

$$
S(\tau)=\left\langle[Z(x+\tau)-Z(x)]^{2}\right\rangle
$$

where $Z(x)$ represents the height values of the contour, and $\tau$ is any interval of the sampling interval.

On the double logarithmic coordinates, the structure function $S(\tau)$ of the $\mathrm{W}-\mathrm{M}$ function can be expressed as follows:

$$
\begin{aligned}
\lg S(\tau)= & \lg \left(\frac{\Gamma(2 D-3) \sin [(2 D-3) \pi / 2]}{(4-2 D) \ln \gamma}\right) \\
& +2(D-1) \lg G+2(2-D) \lg \tau
\end{aligned}
$$

Since the contact of joint surfaces is equivalent to that between an elastic rough surface and a rigid smooth plane, the contour features of two rough surfaces are converted to the equivalent elastic rough surface. Therefore, it is needed to calculate the equivalent fractal parameter for the real contact of two rough surfaces. The structure function of the equivalent elastic rough surface is established by superposing the structure functions of the two contacted surfaces. And the relationship between the structure functions of the equivalent rough surface and the two rough surfaces can be expressed as follows [14]:

$$
S(\tau)=S_{1}(\tau)+S_{2}(\tau)
$$

Notice that $S(\tau)$ represents the structure function of the equivalent elastic rough surface, and $S_{1}(\tau)$ and $S_{2}(\tau)$ respectively represent the structure function of two rough surfaces in contact.

The double logarithmic graphs of structure function of the measured surface profiles are plotted in Figure 6. Then the equivalent fractal parameters $D$ can be derived, which are listed in Table 1.

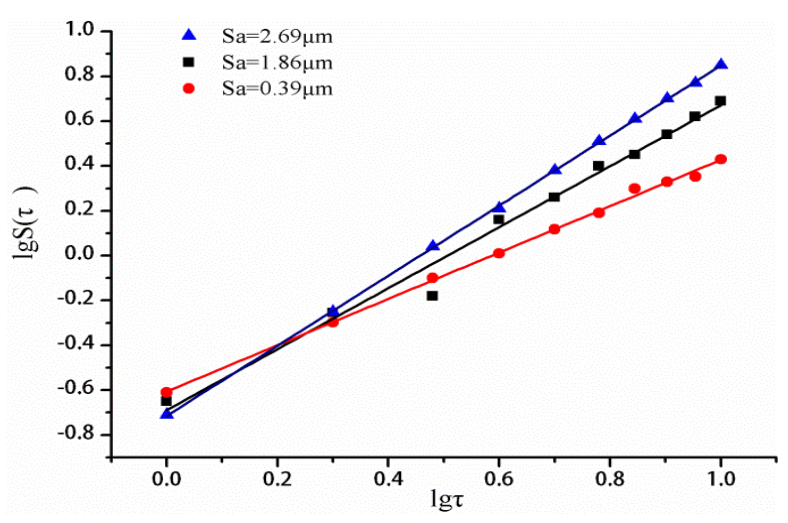

Figure 6 Structure function graph of the surface profiles

Table 1 Equivalent fractal parameter values of the joint surfaces

\begin{tabular}{cccc}
\hline$S a(\mu \mathrm{m})$ & 2.69 & 1.86 & 0.39 \\
\hline$D$ & 1.22 & 1.34 & 1.49 \\
\hline
\end{tabular}

\subsection{Normal contact stiffness of the textured zone}

Surface profiles of specimens are measured by the VKX250 type laser profilometer from KEYENCE, which can be used for three-dimensional measurement and quantitative analysis of rough surface profile and shape in micron and submicron scale. The instrument uses laser source and white-light source to obtain the height information and color information of specimens respectively. According to the material, shape and measurement range of the specimen, three different scanning principles, including laser confocal scanning, 
white light interference and focusing change, can be selected for high-precision measurement. Then the morphology of each texture can be obtained. And the threedimensional model of texture is established based on the morphology of sample textures, as shown in the Figure 7. (a)

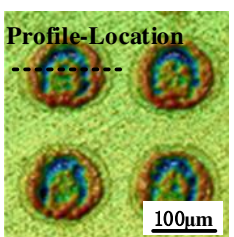

(b)

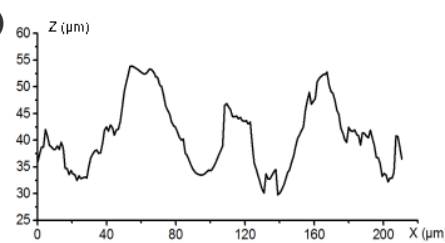

$\mathrm{R}_{2}$

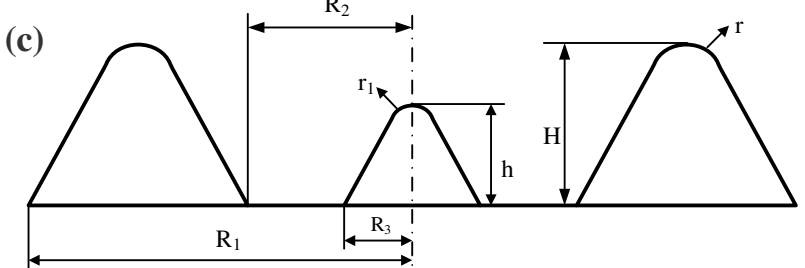

Figure 7 (a) Three-dimensional representation of textured surface; (b)Profile of texture section; (c)Texture model

As shown in Figure 7(c), the texture can be regarded as the melting layer on the outside and the hump on the inside. For the melting layer, it is assumed that the height is $H$, the external diameter at the bottom is $R_{1}$, the internal diameter at the bottom is $R_{2}$, the curvature radius of the top is $r$. For the hump, it is assumed that the height is $h$, the diameter at the bottom is $R_{3}$, the curvature radius of the top is $r_{1}$, and the deformation of the texture is $\delta$. Then the contact area A of textured zone can be given by:

$$
A= \begin{cases}\pi\left(\frac{R_{1}+R_{2}}{2}+\sqrt{2 r \delta-\delta^{2}}\right)^{2}-\pi\left(\frac{R_{1}+R_{2}}{2}-\sqrt{2 r \delta-\delta^{2}}\right)^{2} & \delta \leq r \\ \pi\left[\frac{R_{1}+R_{2}}{2}+l_{1}\right]^{2}-\pi\left[\frac{R_{1}+R_{2}}{2}-l_{1}\right]^{2} & r \leq \delta \leq H-h \\ \pi\left[\frac{R_{1}+R_{2}}{2}+l_{1}\right]^{2}-\pi\left[\frac{R_{1}+R_{2}}{2}-l_{1}\right]^{2}+\pi R_{3}[\delta-(H-h)] & H-h \leq \delta \leq H-h+r_{1} \\ \pi\left[\frac{R_{1}+R_{2}}{2}+l_{1}\right]^{2}-\pi\left[\frac{R_{1}+R_{2}}{2}-l_{1}\right]^{2}+\pi l_{2}^{2} & H-h+r_{1} \leq \delta \leq H \\ \pi\left(R_{1}^{2}-R_{2}^{2}+R_{3}^{2}\right) & \delta_{1} \geq H\end{cases}
$$

where $l_{1}$ is the ring width corresponding to real and truncated contact area for a melting layer, $l_{2}$ is the diameter corresponding to real and truncated contact area for a hump.

$$
l_{1}=2 r+\frac{2(\delta-r)}{h-r}\left(\frac{R_{1}-R_{2}}{2}-r\right)
$$

$$
l_{2}=2 r_{1}+\frac{2\left(R_{3}-r\right)}{h-r}\left(\delta-H+h-r_{1}\right),
$$

The normal contact stiffness $K_{2}$ of the textured zone can be expressed as follows:

$$
K_{2}=\frac{E_{2} A}{A_{n}}
$$

where $E_{2}$ is the elastic modulus of material in textured zone, $A_{\mathrm{n}}$ is the apparent contact area between two contact surfaces.

During processing the surface texturing, the material of the textured zoon is melted, and the material properties is changed. The elastic modulus $E_{2}$ of material in textured zone can be given by

$$
E_{2}=c E_{0}
$$

where $c$ is the variation coefficient of elastic modulus of material in textured zone, and $E_{0}$ is the elastic modulus of the original material.

It is similar to the hardness test. The elastic modulus is measured by IMicro Nanoindenter from KLA along the texture depth direction. The continuous stiffness measurement method is adopted, and the target load is set to $0.25 \mathrm{~N}$. The distance between indentation points in the same column is $30 \mu \mathrm{m}$, as shown in Figure 8. The experimental results show that the elastic modulus of the original surface is $198.3 \mathrm{GPa}$ and that of the textured surface is $225.8 \mathrm{GPa}$. Then the variation coefficient of elastic modulus $\mathrm{c}$ is calculated as 1.14 .

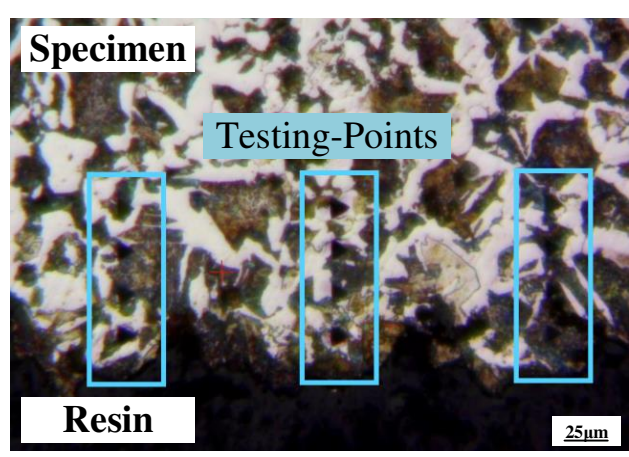

Figure 8 The schematic of elastic modulus test

\section{Experiments}




\subsection{Specimens preparation}

The contact stiffness is mainly related to the material and contact type of joint surfaces. The material of the specimens is R683/IC45e steel, and $E=198.3 \mathrm{GPa}$, $v=0.3$, and dry joint surfaces are considered. Considering the previous research $[29,33]$ and experimental conditions, the sample is designed to study the influence of surface texturing on normal contact stiffness of the joint surfaces, as shown in the Figure 9. The investigated joint surfaces are formed by the contact surfaces between the upper and the lower specimens, which are fabricated by milling. The apparent contact area $A_{\mathrm{n}}$ is $412.3 \mathrm{~mm}^{2}$, and the quality $m$ of the upper and lower specimens is $1.05 \mathrm{~kg}$ in total.

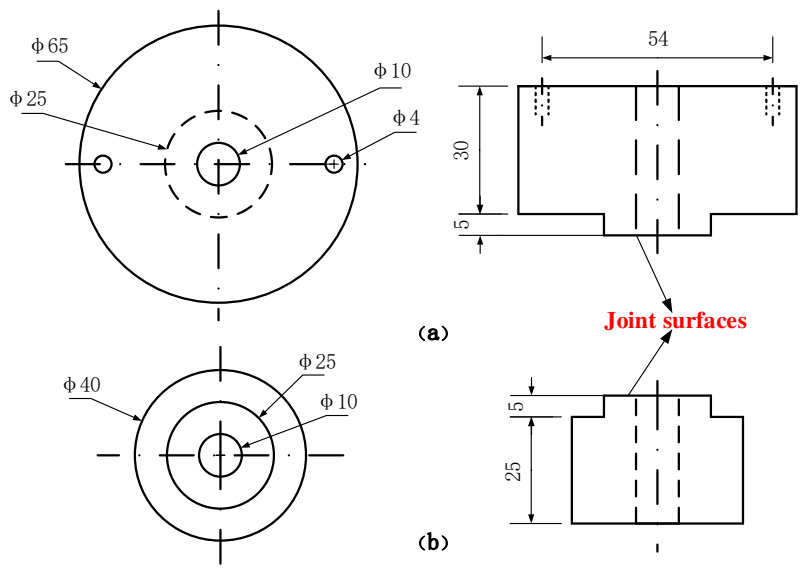

Figure 9 Test specimens (unit, mm): (a) upper specimen, (b) lower specimen

In order to study the effect of surface texturing on the normal contact stiffness of joint surfaces with different processing accuracy, three kinds of specimens were processed. And the surface topography parameters $S a$ are $2.69 \mu \mathrm{m}, 1.86 \mu \mathrm{m}$ and $0.39 \mu \mathrm{m}$, respectively. $S a$ is a roughness evaluation parameter based on the regional morphology, which represents the arithmetic mean deviation of the area topography. Therefore, it can be used to characterize the roughness of the two-dimensional surface of specimens. $S a$ is defined as follows:

$$
S a=(1 / M N) \sum_{i=1}^{M} \sum_{j=1}^{N}\left|Z_{i j}\right|,
$$

where $Z$ is the distance from the point on the contour of the specimens surface to the reference plane, $M$ and $N$ are the sampling points in two directions perpendicular to each other in the evaluation area.
Before processing the surface texture, the surface of the lower specimen is polished in order to eliminate the surface defects caused by the milling including burr, flash, and ploughing. After that, the polished specimens are cleaned in the ultrasonic cleaner to remove the grinding debris. Then, the micro textures in the form of circular dimples are created on the surface of lower specimen by the lsf-20 laser marking machine. The diameter of micro textures is 100 $\mu \mathrm{m}$ and the surface density is $30 \%$, which can effectively reduce wear [20].

During texturing, under the effect of recoil, some liquid and gaseous material will be washed out of the molten pool. Then the part of material falls on surface of the specimens or into the texture, which cools rapidly and forms slag. Since the slag seriously affects the contact characteristics of the joint surfaces, it should be removed. The textured surface is polished with W10 metallographic sandpaper. There are still a lot of wear debris and metal slag on the surfaces of the specimens and inside the textures. Consequently, the ultrasonic cleaning is used to remove these debris and slag. After that, the liquid on the surfaces of the specimens is blown by a dryer. At last, the specimens are numbered and sealed in a clean container.

\subsection{Experimental setup}

The normal contact stiffness of joint surfaces is measured by the method of modal frequency [33-34]. The test rig is shown in Figure 10 [23]. The normal static preload at the joint surfaces is applied by nut and spring, which has a loading range of 0-900 N. The steel balls can ensure as far as possible that the distribution of normal static load at the joint surfaces is uniform. The piezoelectric accelerometers of $1 \mathrm{~A} 116 \mathrm{E}$ from DONGHUA are fixed on the upper specimen symmetrically, which have a resolution of $5 \times 10^{-}$ ${ }^{4} \mathrm{~g}$ and a axial sensitivity of $10.67 \mathrm{mV}\left(\mathrm{m} \cdot \mathrm{s}^{-2}\right)$. The exciting force is excited on the surface of the upper specimen through the impact hammer of LC02 from DONGHUA, which has a range of $0-5000 \mathrm{~N}$ and a sensitivity of 4.24 $\mathrm{pC} / \mathrm{N}$. The schematic view of experiment principle is shown in the Figure 11. The acceleration signal and force signal are input into computer for processing by Dong Hua Dynamic Analysis System(DHDAS). The eigenmode frequency of the test system can be obtained by the analysis of frequency response function of acceleration signal and force signal. According to the Ep. (23), the normal contact stiffness $K_{\mathrm{m}}$ of the specimens can be obtained.

$$
K_{m}=m\left(2 \pi f_{n}\right)^{2} / A_{n},
$$


where $m$ is the quality of upper and lower specimens in total, and $f_{n}$ is the certain eigenmode frequency of the test system.

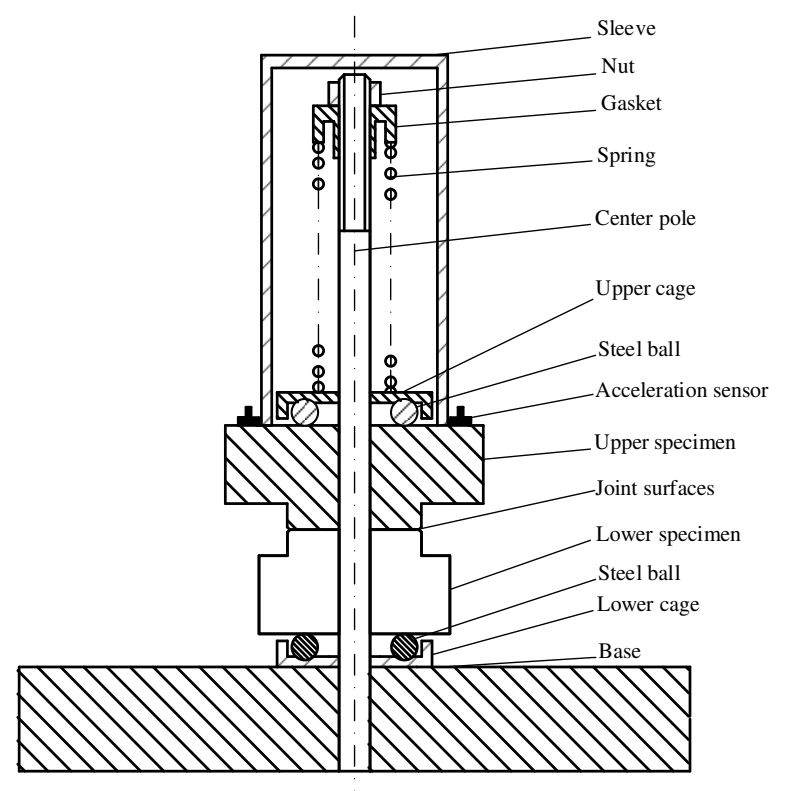

Figure 10 Test rig for normal contact stiffness evaluation

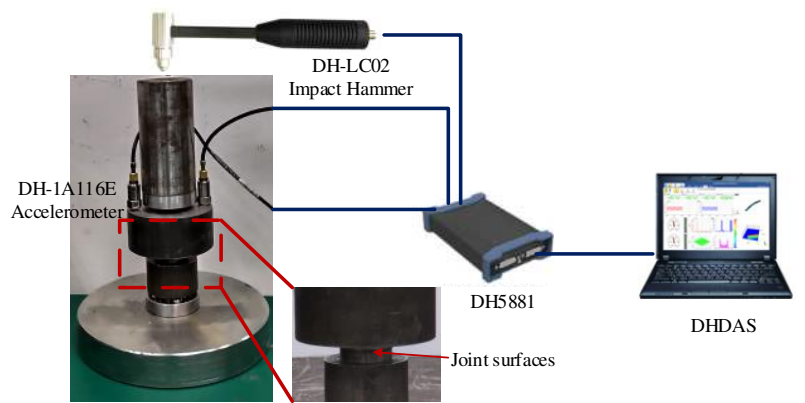

Figure 11 The schematic view of the test

\section{Results and discussions}

According to Eqs (2), (11) and (23), the ExcDec1 was used to fit the experimental data. The results are shown in Figure 12. The values of $R$ (adjusted deviate square) represent the correlation between experimental data and fitting curve, which are usually used to describe the fitting quality of the model. As shown in Figure 12, the parameters $R$ are greater than 0.94 , and the fitting function is appropriate.

For the textured specimens, the predictions of the theoretical model are compared with the experimental data. The consistency between the theoretical predictions and the experimental data is judged by the parameter RNew. The closer the $R N e w$ is to 1 , the better the fitting degree is. The $R N e w$ can be expressed as follows:

$$
R N e w=1-\left(Q / \sum y^{2}\right)^{\frac{1}{2}},
$$

$$
Q=\sum\left(y-y^{*}\right)^{2}
$$

where $y$ is the experimental value and $y^{*}$ is the theoretical prediction value, and $Q$ is the residual sum of squares.

According to Ep. (24), the values of parameter RNew for the specimens are recorded in Table 2.

Table 2 Parameter $R N e w$ for the specimens

\begin{tabular}{cccc}
\hline$S a(\mu \mathrm{m})$ & 0.39 & 1.86 & 2.69 \\
\hline$R N e w$ & 0.89 & 0.90 & 0.91 \\
\hline
\end{tabular}

It can be seen from the Table 2 that the values of parameter $R N e w$ are all about 0.9 for different specimens. Therefore, it can be concluded that the theoretical results of the proposed model are consistent with the experiment data.

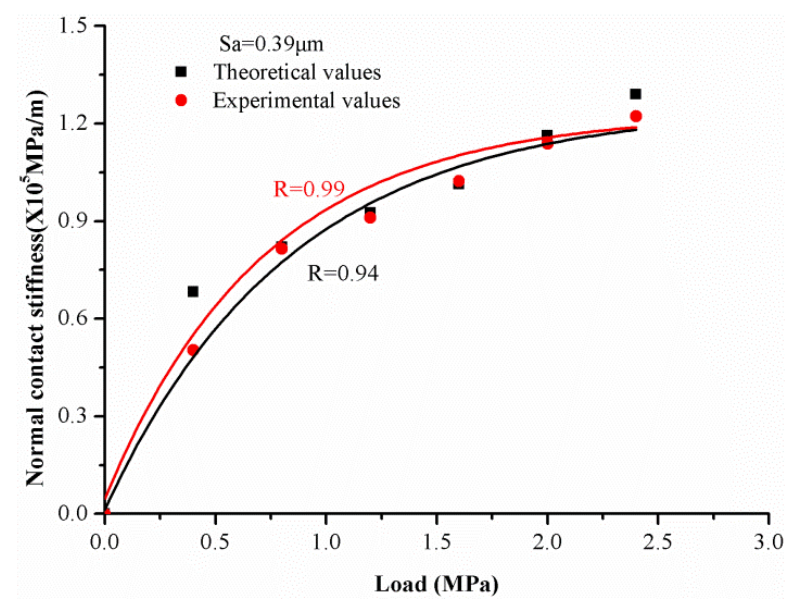

(a) The joint surfaces with $S a=0.39 \mu \mathrm{m}$ 


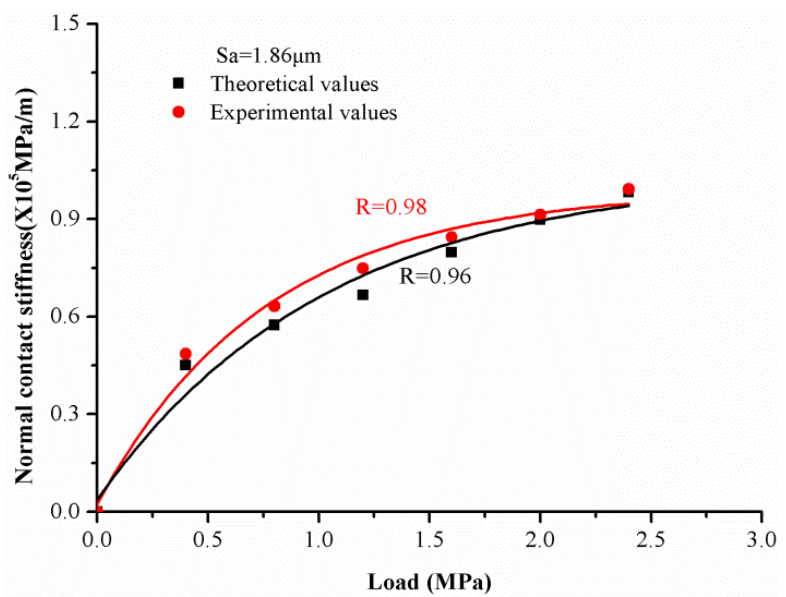

(b) The joint surfaces with $S a=1.86 \mu \mathrm{m}$

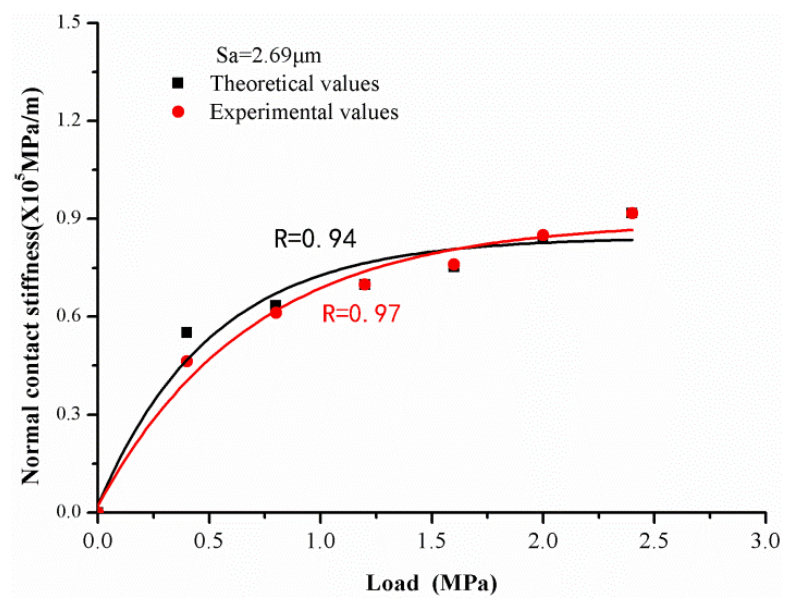

(c) The joint surfaces with $S a=2.69 \mu \mathrm{m}$

Figure 12 Comparison of theoretical predictions and experimental data for different joint surface

For the textured specimens, the relationships of both the predictions of proposed model and the experimental data on the normal load for different rough surfaces are shown in Figure 12. As the surface roughness decreases, the normal contact stiffness of joint surfaces gradually increases for a fixed load. The reason is that when two rough surfaces are in contact, the normal contact stiffness of joint surfaces depends on the real contact area, and the real contact area gradually increases as the roughness of rough surface decreases. Also, it can be seen from Figure 12 that the theoretical predictions are consistent with the experimental data, and the estimation model for normal contact stiffness of joint surfaces with surface texturing is appropriate.

For the joint surfaces with different roughness, the influence of surface texturing on normal contact stiffness is different. For the joint surfaces with $S a=2.69 \mu \mathrm{m}$, when the load is less than 1.3 MPa, the surface texturing reduces the normal contact stiffness of the joint surfaces. As the load increases, the difference first increases and then decreases. When the load is greater than 1.3 $\mathrm{MPa}$, the surface texturing increases the normal contact stiffness of joint surfaces on the contrary. As the load increases, the increasing difference is getting larger. When the load is 2.4 $\mathrm{MPa}$, the normal contact stiffness of joint surfaces with surface texturing is increased by $15 \%$, as shown in Figure 13.

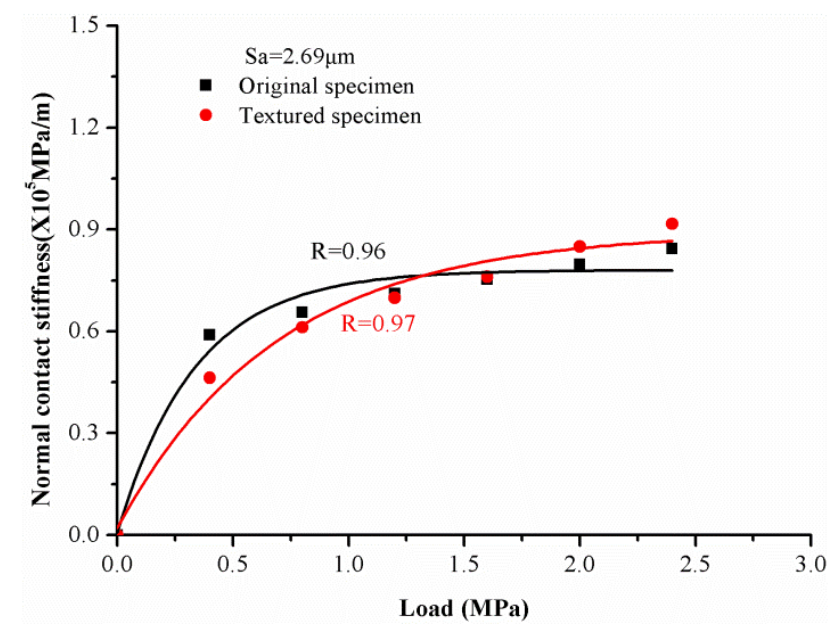

Figure 13 Experimental values of the joint surfaces with $S a=2.69 \mu \mathrm{m}$

For the joint surfaces with $S a=1.86 \mu \mathrm{m}$, when the load is less than $1.8 \mathrm{Mpa}$, the surface texturing reduces the normal contact stiffness of joint surfaces. With the increase of the load, the variety of the difference is similar to that of the joint surfaces with $S a=2.69 \mu \mathrm{m}$. Then, when the load continues to increase, the surface texturing on the contrary increases the normal contact stiffness of joint surfaces. The growth difference is relatively slow compared with that of the joint surfaces with $S a=2.69 \mu \mathrm{m}$. When the load is 2.4 $\mathrm{MPa}$, the normal contact stiffness of joint surfaces with surface texturing is increased by $8 \%$, as shown in Figure 14.

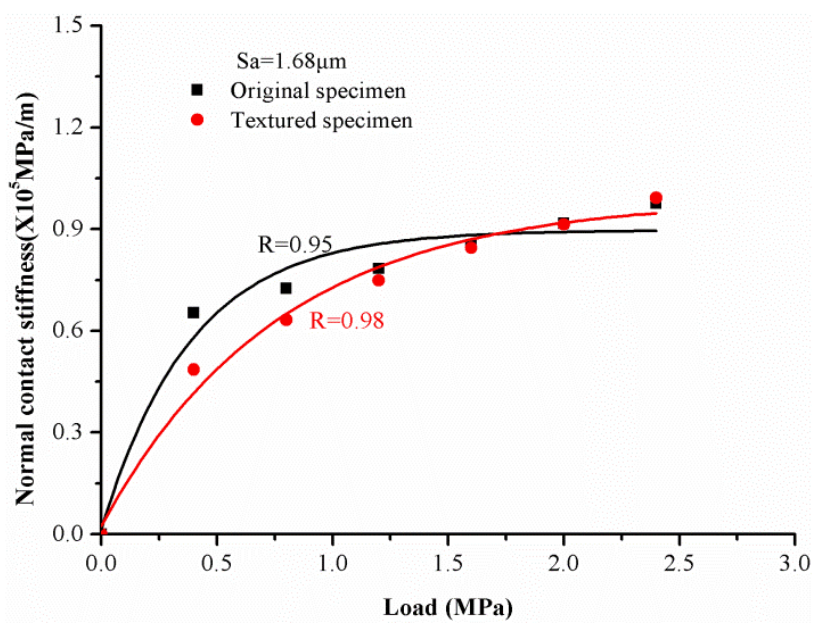

Figure 14 Experimental values of the joint surfaces with $S a=1.86 \mu \mathrm{m}$ 
For the joint surfaces with $S a=0.39 \mu \mathrm{m}$, the surface texturing obviously reduces the normal contact stiffness of joint surfaces. As the load increases, the difference gradually increases. When the load is greater than $1.2 \mathrm{MPa}$, the normal contact stiffness of joint surfaces with surface texturing is reduced by $20 \%$, as shown in Figure 15.

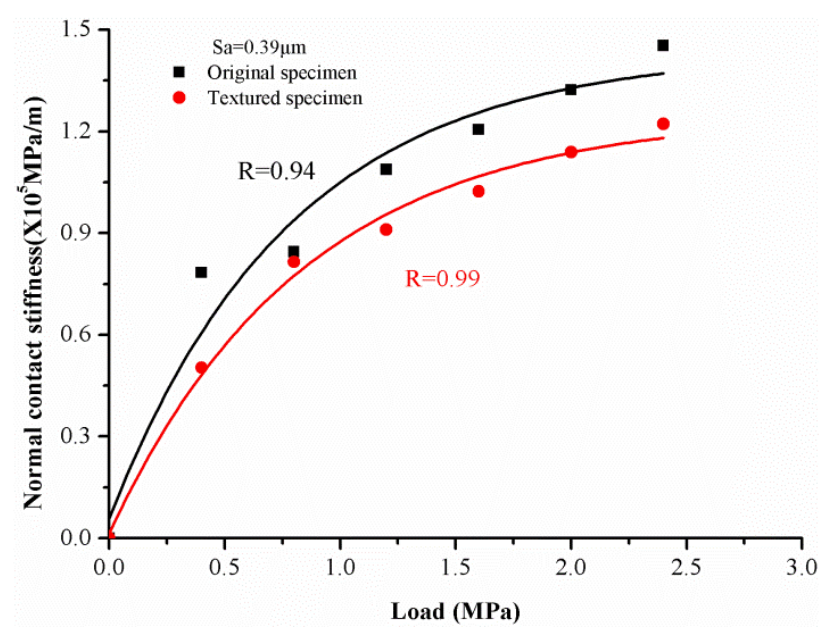

Figure 15 Experimental values of the joint surfaces with $S a=0.39 \mu \mathrm{m}$

Essentially, the influence of surface texturing on the normal contact stiffness of the joint surfaces is that part of the original contact area is replaced by the textured zone. Because the average height of textures is larger than that of surface asperities. When the normal load is applied the textures first yields contact deformation. As the normal load increases, the amount of deformation gradually increases, and then the surface asperities also begin to yield contact deformation. Therefore, when the normal load is small, the textured zone is mainly involved in the contact deformation, which is equivalent to the textured zone replacing the entire joint surfaces. This is the reason that the normal contact stiffness of textured specimens is smaller than that of the original specimens in the initial stage of contact deformation.

For the different joint surfaces, as $S a$ increases, the real contact area gradually decreases; however, the contact area of textured zone is constant. When $S a>1.86 \mu \mathrm{m}$, the normal contact stiffness of the original joint surfaces is relatively small. And the normal contact stiffness of the textured zone is greater than that of the replaced original joint surfaces. Therefore, the overall contact stiffness of the joint surfaces is increased. Otherwise, when $S a$ is relatively small, the normal contact stiffness of the textured zone is smaller than that of replaced original joint surfaces. Therefore, the overall contact stiffness of the joint surfaces is reduced.

\section{Conclusions}

(1) Considering surface morphology and material properties, the theoretical model is proposed in order to study the influence of surface texturing on the normal contact stiffness of the joint surfaces. Surface topography of the rough surface is described based on the fractal theory. The structure function method was used to calculate the fractal dimension of the rough surface profiles.

(2) The theoretical predictions are consistent with the experimental data through the parameter $R \mathrm{New}$, which indicates that the theoretical model for normal contact stiffness of the joint surfaces with surface texturing is appropriate.

(3) The normal contact stiffness of textured specimens is smaller than that of the original specimen in the initial stage of contact deformation. For the joint surfaces with $S a>2.69 \mu \mathrm{m}$, the normal contact stiffness can be effectively increased through proper surface texturing.

\section{Declaration}

\section{Acknowledgements}

The authors sincerely thanks to Professor Haihong Huang of Hefei University of Technology for his critical discussion and reading during manuscript preparation.

\section{Funding}

Supported by National Natural Science Foundation of China (Grant No. 51635010, 51722502 and 51675155).

\section{Authors' Contributions}

$\mathrm{HH}$ was in charge of the whole trial; CY wrote the manuscript; DZ assisted with writing manuscript; JF assisted with sampling and laboratory analyses. All authors read and approved the final manuscript.

\section{Competing Interests}

The authors declare that they no competing interests.

\section{Consent for publication \\ Not applicable}

\section{Ethics approval and consent to participate}

Not applicable

\section{References}

[1] Y. T. Ai, C. Yang, J. Tian, and Z. Wang, "Effects of NonLinear Characteristics of Joint Surface on Dynamic Performance of Flange Bolt Connection," Tuijin Jishu/Journal of Propulsion Technology, vol. 39, no. 9, pp. 2068-2074, 2018. 
[2] B. Singh and B. K. Nanda, "Identification of damping mechanism in layered and welded structures," (in English), Int J Mech Sci, vol. 63, no. 1, pp. 37-47, Oct 2012.

[3] D. D. Quinn, "Modal analysis of jointed structures," Journal of Sound and Vibration, vol. 331, no. 1, pp. 81-93, 2012.

[4] J. D. Miller and D. D. Quinn, "A two-sided interface model for dissipation in structural systems with frictional joints," (in English), Journal of Sound and Vibration, vol. 321, no. 1-2, pp. 201-219, Mar 202009.

[5] J. A. Greenwood and J. B. P. P. Williamson, "Contact of Nominally Flat Surfaces," Proceedings of the Royal Society of London, vol. 295, no. 1442, pp. 300-319, 1966.

[6] B. Bhushan and A. Majumdar, "Elastic-plastic contact model for bifractal surfaces," Wear, vol. 153, no. 1, pp. 5364, 1992.

[7] S. Wang and K. Komvopoulos, "A Fractal Theory of the Interfacial Temperature Distribution in the Slow Sliding Regime: Part II-Multiple Domains, Elastoplastic Contacts and Applications," Journal of Tribology, vol. 116, no. 4, p. 824, 1994.

[8] Y. Zhao, D. M. Maietta, and L. Chang, "An Asperity Microcontact Model Incorporating the Transition From Elastic Deformation to Fully Plastic Flow," Journal of Tribology, vol. 122, no. 1, p. 86, 2000.

[9] S. Y. Jiang, Y. J. Zheng, and H. Zhu, "A Contact Stiffness Model of Machined Plane Joint Based on Fractal Theory," (in English), J Tribol-T Asme, vol. 132, no. 1, Jan 2010.

[10] Y. Zheng, Z. Hou, and Y. Rong, "The study of fixture stiffness - Part II: contact stiffness identification between fixture components," The International Journal of Advanced Manufacturing Technology, vol. 38, no. 1-2, pp. 19-31, 2007.

[11] Y. Sun, H. Xiao, J. Xu, and W. Yu, "Study on the normal contact stiffness of the fractal rough surface in mixed lubrication," ARCHIVE Proceedings of the Institution of Mechanical Engineers Part J Journal of Engineering Tribology 1994-1996 (vols 208-210), p. 135065011875874, 2018.

[12] M. Ciavarella, J. A. Greenwood, and M. Paggi, "Inclusion of "interaction" in the Greenwood and Williamson contact theory," Wear, vol. 265, no. 5-6, pp. 729-734, 2008.

[13]M. Belhadjamor, S. Belghith, S. Mezlini, and M. E. Mansori, "Numerical study of normal contact stiffness: Non-Gaussian roughness and elastic-plastic behavior," ARCHIVE Proceedings of the Institution of Mechanical Engineers Part
J Journal of Engineering Tribology 1994-1996 (vols 208210), p. $135065011989352,2019$.

[14] R. WANG, "Investigation of Contact Stiffness Model for Joint Surfaces Based on Domain Expansion Factor and Asperity Interaction," Journal of Mechanical Engineering vol. 54, no. 19, p. 88, 2018.

[15] Y. Zhang, H. Lu, X. Zhang, H. Ling, and Z. Guo, "A normal contact stiffness model of machined joint surfaces considering elastic, elasto-plastic and plastic factors," ARCHIVE Proceedings of the Institution of Mechanical Engineers Part J Journal of Engineering Tribology 19941996 (vols 208-210), vol. 234, no. 7, p. 135065011986780 , 2019.

[16] L. Zhu, H. Li, and W. Wang, "Research on rotary surface topography by orthogonal turn-milling," Int $J$ Adv Manuf Tech, vol. 69, no. 9-12, pp. 2279-2292, 2013.

[17] C. Zhai, Y. Gan, D. Hanaor, G. Proust, and D. Retraint, "The Role of Surface Structure in Normal Contact Stiffness," Experimental Mechanics, vol. 56, no. 3, pp. 359-368, 2015.

[18] Z. W. Guo, C. Q. Yuan, X. Q. Bai, and X. P. Yan, "Experimental Study on Wear Performance and Oil Film Characteristics of Surface Textured Cylinder Liner in Marine Diesel Engine," (in English), Chin J Mech Eng-En, vol. 31, no. 1, p. 52, Dec 2018.

[19] S. Swirad, "Surface Texture Analysis after Hydrostatic Burnishing on X38CrMoV5-1 Steel," (in English), Chin J Mech Eng-En, vol. 32, no. 1, Nov 72019.

[20] Q. Chang, "Tribological Influence of Laser Surface Textures on 45 Steel under Dry Sliding," Journal of Mechanical Engineering, vol. 53, no. 3, p. 148, 2017.

[21] M. Arghir, F. Billy, G. Pineau, J. Frene, and A. Texier, "Theoretical analysis of textured "Damper" annular seals," (in English), J Tribol-T Asme, vol. 129, no. 3, pp. 669-678, Jul 2007.

[22] S. Medina, D. Nowell, and D. Dini, "Analytical and Numerical Models for Tangential Stiffness of Rough Elastic Contacts," Tribology Letters, vol. 49, no. 1, pp. 103-115, 2012.

[23] Z. Fuadi, T. Takagi, H. Miki, and K. Adachi, "An experimental method for tangential contact stiffness evaluation of contact interfaces with controlled contact asperities," (in English), P I Mech Eng J-J Eng, vol. 227, no. 10, pp. 1117-1128, Oct 2013. 
[24] Z. Yi and S. Xi, "Experimental Investigation on Effect of Surface Texture on Tangential Contact Parameters of Mechanical Joint Interface," Machine Building \& Automation, 2014.

[25]M. Belhadjamor, S. Belghith, S. Mezlini, and M. El Mansori, "Numerical study of normal contact stiffness: Non-Gaussian roughness and elastic-plastic behavior," Proceedings of the Institution of Mechanical Engineers, Part J: Journal of Engineering Tribology, vol. 234, no. 9, pp. 1368-1380, 2019.

[26] W. Huang, Y. Huang, and H. A. University, "Microstructure and Property of 45 Steel by Laser Melting-hardening Strengthening," Hot Working Technology, 2017.

[27] Y. Qu, J. Zhou, L. Zhao, R. Zhang, and Z. Tian, "Fiber laser remelting technology of 45 steel," Hanjie Xuebao/Transactions of the China Welding Institution, vol. 36, no. 7, pp. 59-62, 2015.

[28] Q. C. Sun, X. Liu, X. K. Mu, and Y. C. Gao, "Estimation for normal contact stiffness of joint surfaces by considering the variation of critical deformation," (in English), Assembly Autom, vol. 40, no. 3, pp. 399-406, Jan 22020.

[29] D. Guan, L. Jing, H. H. Hilton, J. J. Gong, and Z. W. Yang, "Tangential contact analysis of spherical pump based on fractal theory," (in English), Tribol Int, vol. 119, pp. 531-538, Mar 2018.

[30] A. Majumdar and B. Bhushan, "Fractal Model of ElasticPlastic Contact Between Rough Surfaces," J Tribol Trans Asme, vol. 113, no. 1, pp. 1-11, 1991.

[31] H. Xiao, Y. Sun, and Z. Chen, "Fractal modeling of normal contact stiffness for rough surface contact considering the elastic-plastic deformation," Journal of the Brazilian Society of Mechanical Sciences and Engineering, vol. 41, no. $1,2018$.

[32] J. J. Wu, "Characterization of fractal surfaces," Wear, vol. 239, no. 1, pp. 36-47, 2000.

[33] P. Ren, L. Wang, and C. Wang, "Experimental Study on Normal Dynamic Contact Stiffnesses of Trail Interfaces under Lubrications," Zhongguo Jixie Gongcheng/china Mechanical Engineering, vol. 29, no. 7, pp. 811-816, 2018.

[34] J. D. Li, Y. S. Zhu, K. Yan, X. Y. Yan, Y. W. Liu, and J. Hong, "Research on the axial stiffness softening and hardening characteristics of machine tool spindle system," (in English), Int J Adv Manuf Tech, vol. 99, no. 1-4, pp. 951-963, Oct 2018.

\section{Biographical notes}

Chao-Chao Yin, is currently a $\mathrm{PhD}$ candidate at Key Laboratory of Green Design and Manufacturing of Mechanical Industry, Hefei University of Technology, Hefei, China.

Tel:+86-18297925175;

E-mail: 2016110111@mail.hfut.edu.cn

Hai-Hong Huang, is currently a professor, PhD Tutor at School of Mechanical Engineering, Hefei University of Technology, Hefei, China.

Tel:+86-13955138301; E-mail:huanghaihong@hfut.edu.cn

Dan Zhou, is currently an associate professor at School of Mechanical Engineering, Hefei University of Technology, Hefei, China.

E-mail: zhoudan80@126.com

Zhi-Feng Liu, is currently a professor, PhD Tutor at School of Mechanical Engineering, Hefei University of Technology, Hefei, China.

E-mail: zhfliuhfut@126.com 